\title{
Latent functional diversity may accelerate microbial community responses to environmental fluctuations
}

\author{
Thomas P. Smith ${ }^{1, *}$, Shorok Mombrikotb ${ }^{1}$, Emma Ransome ${ }^{1}$, Dimitrios-Georgios \\ Kontopoulos ${ }^{1}$, Samraat Pawar ${ }^{1}$, and Thomas Bell ${ }^{1}$ \\ ${ }^{1}$ Department of Life Sciences, Imperial College London, Silwood Park, \\ Ascot, Berkshire SL5 7PY, UK \\ ${ }^{*}$ To whom correspondence should be addressed: \\ thomas.smith1@imperial.ac.uk
}

\begin{abstract}
Whether and how whole ecological communities can respond to climate change remains an open question. With their fast generation times and abundant functional diversity, microbes in particular harbor great potential to exhibit community-level adaptation through a combination of strain-level adaptation, phenotypic plasticity, and species sorting. However, the relative importance of these mechanisms remains unclear. Here, through a novel laboratory experiment, we show that bacterial communities can exhibit a remarkable degree of community-level adaptability through a combination of phenotypic plasticity and species sorting alone. Specifically, by culturing soil communities from a single location at six temperatures between $4^{\circ} \mathrm{C}$ and $50^{\circ} \mathrm{C}$, we find that multiple strains well adapted to different temperatures can be isolated from the community, without immigration or strain-level adaptation. This is made possible by the ability of strains with different physiological and life history traits to "switch on" under suitable conditions, with phylogenetically distinct $K$-specialist taxa favoured under cooler conditions, and $r$-specialist taxa in warmer conditions. Our findings provide new insights into microbial community adaptation, and suggest that microbial community function is likely to respond rapidly to climatic fluctuations, through changes in species composition during repeated community assembly dynamics.
\end{abstract}

\section{Introduction}

Temperature has a pervasive influence on microbial communities because of its impact on microbial physiology and fitness $[1,2,3]$. Microbes are drivers of key ecosystem processes: they play a central role in global biogeochemical cycles and are tightly linked to the wider ecosystem due to their role as pathogens, mutualists, as a food source for higher trophic levels, and in nutrient cycling. There is therefore great interest in understanding how temperature impacts microbial community dynamics, and how those impacts percolate to the surrounding ecosystem [4].

Temperature varies at multiple scales, from seconds (i.e. sun/shade), to daily and seasonal temperature fluctuations, as well as longer-term changes including anthropogenic climate warming and temperature changes over geological time scales. As temperature changes over time and space, microbial fitness is expected to vary, both in the short term through phenotypic or physiological plasticity, and long term through genetic adaptation. There is a large body of work profiling the thermal niches of microbial taxa, by observing growth rates (a proxy for fitness) across a range of temperatures [5, 3]. The relationship between growth rate and temperature is typically unimodal, with $\mu_{\max }$ defined as the maximum growth rate across the temperature range, and $T_{\mathrm{opt}}$ (the optimal growth temperature) defined as the temperature at which growth rate is maximum. $T_{\mathrm{opt}}$ is expected to match the prevailing environmental conditions experienced by the organism $[6,7]$, within some tolerance margins to account for temperature fluctuations. This parameter evolves gradually through evolutionary time [8], potentially through structural changes to enzymes that alter their melting temperatures [9]. However, bacteria are also known to be able to rapidly adapt to new temperatures in adaptive evolution experiments (through shifts in $T_{\mathrm{opt}}$ ) [10,11, 12], although the underlying molecular mechanisms are unclear. 
bioRxiv preprint doi: https://doi.org/10.1101/2021.04.14.439774; this version posted April 14, 2021. The copyright holder for this preprint made available under aCC-BY-NC 4.0 International license.

While there has been substantial research profiling the thermal performance of individual taxa, relatively little is known about how taxa respond to shifting temperature in a community context. Studies of individual populations that have been artificially isolated from the surrounding community might give misleading results because interactions within communities can constrain or accelerate adaptive evolution [13]. Although bacterial community dynamics are strongly influenced by temperature [14], there are few experimental studies that have tracked the thermal performance of populations within a community. Taxa that are embedded within communities can respond to shifting temperature in two ways, which are not mutually exclusive:

(1) Acclimation/adaptation: the constituent populations can acclimate (short time scales) or adapt (long time scales) to the new temperature. For example, they may up-regulate genes that are more appropriate for the new temperature, or alter the fatty acids used in building cell walls to the local temperature conditions [15]. Many investigations into changes in ecosystem function with temperature change have identified the ways by which microbial communities may acclimate or adapt to climate warming $[16,17,18,19]$.

(2) Sorting: the change in temperature could result in selection acting on pre-existing genetic variation within the community. Under this scenario, taxa that were not adapted to the temperature would become inactive (short time scales) or extinct (long time scales), and would be replaced by taxa with preadaptations to the new temperature. Several studies have shown that microbial community composition responds rapidly to warming [20,21], and such changes in community composition are likely to have an important impact on how ecosystem processes respond to climate change $[22,18,19,23]$. While the importance of species sorting in microbial communities has been relatively well studied [24, 25, 26], we currently lack a mechanistic understanding of how variation in population-level thermal physiology influences the environmental filtering process.

Identifying the relative importance of these two mechanisms would allow us to pinpoint the main constraints on microbial community responses to temperature. However, experiments that test these mechanisms are complicated by the extraordinary taxonomic diversity of microbial communities. Sequencing studies have shown a vast genetic diversity of microbes [27], with thousands of distinct microbial taxa present in small environmental samples [28] most of which are found at low abundance [29]. Observational studies have provided support for the idea that bacteria are often found well outside of their thermal niche. For example, studies of the biogeography of thermophilic bacteria have shown that they can be found in benign environments under thermal conditions that prevent their growth, such as cold ocean beds and cool soils [30, 31,32], demonstrating they are widely dispersed outside of their thermal niche. This is likely due either to taxa with large niche widths not being 'sorted' by environmental conditions and persisting within the community regardless of environmental change [25], or to taxa having ways to 'escape' sorting (such as through spore formation). Therefore, the high levels of biodiversity within microbial communities may play a significant role, providing a reservoir of 'latent' diversity (i.e. cells that are present but inactive) that is readily available for sorting. In order to understand the effects of climate change on community composition and thus ecosystem processes, it is therefore important to understand the processes driving community assembly and succession. This involves understanding the amount of phenotypic diversity present at local levels and thus whether species sorting and environmental filtering are likely to drive community change with or without the effects of dispersal and immigration. For example, while many microbial taxa may have the capacity to rapidly acclimate/adapt to a shift in temperature, this capacity would not be ecologically relevant if they are swiftly out-competed by pre-adapted species.

A further consideration is whether differing temperature conditions may favour communities with different life-history strategies. For example, the frequency and magnitude of temperature fluctuations may influence the life history strategies of the taxa in the community [33, 34], which will in turn alter the importance of sorting vs. acclimation/adaption. Environments that have frequent rapid changes in temperature may be dominated by thermal generalists that perform well across a range of temperature conditions [33, 35], and would be able to rapidly adjust their metabolism as temperatures fluctuate (i.e. via acclimation). However, there are theorised to be costs associated with a generalist strategy, resulting in lower maximum growth rates compared to specialists [36, 7] (although evidence for this trade-off may be lacking [37]). Alternatively, frequent, large changes in temperature may favour fast-growers that can rapidly take advantage of the new thermal conditions before they change again. Conversely, constant environments may select for carrying-capacity specialists ( $K$ strategists) rather than growthrate specialists ( $r$ strategists) [38]. The relative importance of these mechanisms is likely to depend upon the temporal scale of fluctuations. The relatively short generation times of microbes means that we may expect them to experience temperature fluctuations on a substantially greater magnitude at inter- 
rather than intra-generational scales [37], potentially favouring specialists rather than generalists [33]. Understanding differences in growth strategies present in bacterial communities is therefore important for understanding community change due to species sorting. Temperature change may drive increased abundance of specialists adapted to different temperatures, or alternatively the community response may be mediated by generalists which perform well across a range of environmental conditions. Furthermore, whether temperature affects the ratio of $r$ to $K$ specialists is likely to have important impacts on the community response to climate change.

Here we investigate how species sorting and latent functional diversity can result in reorganization of bacterial communities to cope with changes in environmental temperature. We performed a species sorting experiment to assess the thermal niche breadth of bacteria within a single soil patch. We compared thermal optima of cultivated taxa to their isolation conditions to understand whether species sorting processes are likely to lead to changes in the relative abundances of taxa based on their thermal adaptations. We tested whether particular clades of bacteria may be deferentially selected for under different thermal conditions, through a phylogenetic analysis to understand the evolutionary history of $T_{\mathrm{opt}}$ in our isolated strains. Finally, by comparing measures of growth, respiration, ATP, carrying capacity and thermal niches, we were able to investigate variation in the trade-offs in growth strategies between these taxa across the temperature gradient. We use these results to unpack the mechanistic, individual-level basis of community-level changes with temperature.

\section{Materials and Methods}

We performed a species sorting experiment to investigate how microbial communities respond to a shift in temperature (Fig. 1). After each sorting experiment we characterised each isolate's thermal performance curve (TPC) of maximal growth rate. We used these TPCs to determine whether there are species present ready to colonise a new temperature niche, should it become available, by measuring how the temperature of peak growth rate $\left(T_{\mathrm{opt}}\right)$ varied with isolation temperature. We performed a phylogenetic analysis to identify whether deep evolutionary differences can predict which taxa are favored by sorting at different temperatures. To understand variation in thermal generalisation vs specialisation and population growth strategy across the temperature gradient, we quantified thermal niche widths of isolates using the TPC of maximal growth rate, and whether they were $r$ - or $K$-strategists by measuring available cellular metabolic energy (ATP), respiration rates, and yield at population steady state (carrying capacity).

\section{Species sorting experiment}

Soil cores were taken from a single site in Nash's field (Silwood Park, Berkshire, UK) in June 2016. Six cores were taken from the top $10 \mathrm{~cm}$ of soil, using a $1.5 \mathrm{~cm}$ diameter sterile corer. Ambient soil temperature at time of sampling was $19.4^{\circ} \mathrm{C}$. The cores were maintained at different temperatures in the laboratory $\left(4^{\circ} \mathrm{C}, 10^{\circ} \mathrm{C}, 21^{\circ} \mathrm{C}, 30^{\circ} \mathrm{C}, 40^{\circ} \mathrm{C}, 50^{\circ} \mathrm{C}\right)$ for four weeks to allow species sorting to occur at those temperatures. The soil was re-hydrated periodically with autoclaved Milli- $\mathrm{Q}$. water during incubation. During this period, in each microcosm, we expected certain taxa to be excluded as the temperature lay outside their thermal niche (environmental filtering), and thermal acclimation of the survivors to occur. Furthermore, we expect that the 4 -week incubation period is likely sufficient time for changes in species interactions, and therefore interaction-driven sorting to occur in addition to the environmental filtering and acclimation. Next, to isolate strains, soil from each core was washed with PBS, plated onto R2 agar and incubated again, at both their respective temperature treatments ("sorting temperature") and at $22^{\circ} \mathrm{C}$ ("standard temperature"), until bacterial colonies formed. In this step, we expect further species sorting to occur, where strains with the highest growth rates at that given temperature form colonies the earliest. The additional isolation of strains at a fixed standard temperature from each of the subcommunities from 6 sorting temperatures was done to identify whether the 4 -week period had indeed resulted in sorting for thermally adapted taxa. If strains adapted to the standard temperature appeared, it would indicate that species sorting was incomplete in the sense that higher or lower temperature treatments had not eliminated them. We also investigated whether any strains that appeared at the standard temperature had undergone thermal adaptation by quantifying their thermal performance curves (explained below). The time-frame for colony appearance on the agar plates differed between temperature treatments, ranging from $\sim 10$ days at $4^{\circ} \mathrm{C}$ to $\sim 1.5$ days at $50^{\circ} \mathrm{C}$ ). Morphologically different colonies were then isolated from each of the 6 sorting temperature and 6 standard temperature plates on R2 agar by streak-plating, before being frozen as glycerol stocks (Fig. 1). In total, 74 strains were isolated in this way. 
Sampling

B

Species

Sorting

(4 weeks)

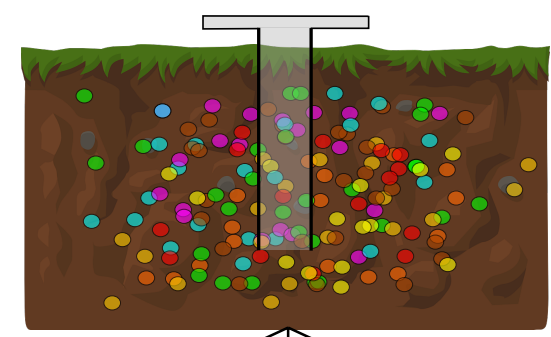

C

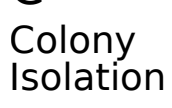

D

Traits
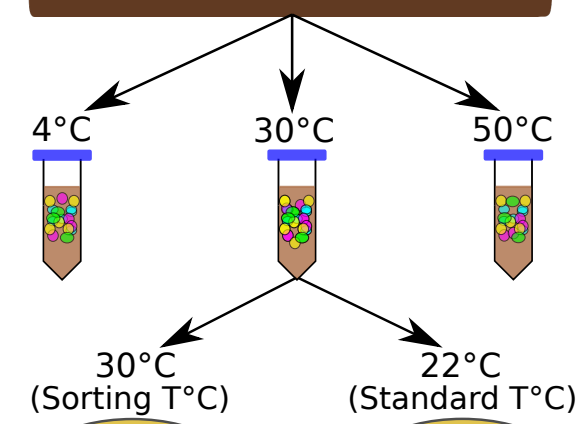

\section{Isolation}
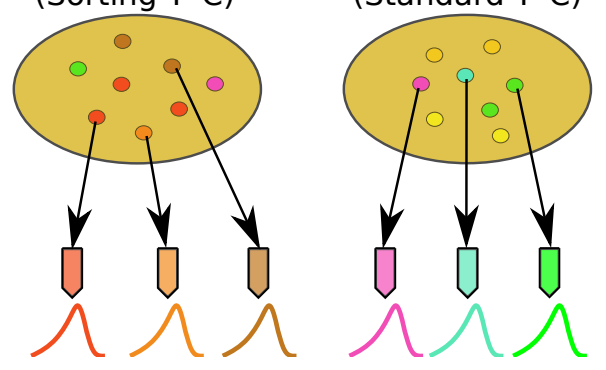

Figure 1: The species sorting experiment. A: Different bacterial taxa (coloured circles) sampled from the soil community. B: Samples maintained at $4,10,21,30,40$ and $50^{\circ} \mathrm{C}$ (only three temperatures shown for illustration), allowing species sorting for 4 weeks. C: Soil washes from each core plated out onto agar and grown at both the sorting temperature and $22^{\circ} \mathrm{C}$ (Standard temperature) to allow further species sorting and facilitate isolation (next step). D: The 6 most abundant (morphologically different) colonies from each plate were picked, streaked and isolated, followed by quantification of physiological and life history traits.

\section{Taxonomic identification}

$16 \mathrm{~S}$ rDNA sequences were used to identify the isolates. Raw sequences were first trimmed using Geneious 10.2.2 (https://www.geneious.com), BLAST searches were then used to assign taxonomy to each trimmed sequence. Where all BLAST hits above $97 \%$ sequence similarity were to the same species, that species was assigned to the isolate. In cases where no species hits were above $97 \%$ sequence similarity, or there were multiple different species hits above $97 \%$ similarity, taxonomy was assigned only to genus level.

\section{Quantifying physiological and life-history traits}

Growth, respiration, and ATP content

We measured growth rate and respiration rate simultaneously across a range of temperatures for each isolate to construct its acute thermal performance curves (TPCs) for these two traits. ATP content of the entire cell culture was also measured at the start and end of the growth assay. Strains were revived from glycerol stocks into fresh LB broth and incubated to carrying capacity at the temperature of the subsequent experiment. This was the acclimation period, which typically took between $72 \mathrm{hrs}$ (warmest temperatures) to $500 \mathrm{hrs}$ (coldest temperature). Biomass abundance was determined by $\mathrm{OD}_{600}$ - optical density measurements at $600 \mathrm{~nm}$ wavelength. Then, prior to each growth-respiration assay, the strains were diluted 1:100 in LB, pushing them into a short lag phase before exponential growth started again (also tracked by $\mathrm{OD}_{600}$ measurements). The exponentially-growing cultures were subsequently spun down at 8,000rpm for 5 minutes to pellet the cells and then re-suspended in fresh LB to obtain $400 \mu$ l culture at a final $\mathrm{OD}_{600}$ of $\sim 0.2-0.3$. This yielded cells primed for immediate exponential growth without a 
lag-phase. These cultures were serially diluted in LB (50\% dilutions) three times, producing a range of starting densities of growing cells. $100 \mu \mathrm{l}$ sub-cultures of each strain were taken and $\mathrm{OD}_{600}$ was tracked in a Synergy HT microplate reader (BioTek Instruments, USA) to ensure that cells were indeed in exponential growth. Initial ATP measurements were made using the BacTiter-Glo assay (see below for details) and cell counts were taken, using a BD Accuri C6 flow cytometer (BD biosciences, USA). Cells were then incubated with a MicroResp ${ }^{\mathrm{TM}}$ plate to capture cumulative respiration (see below for details of the MicroResp system) at the experimental temperature and allowed to continue growing for a short period of time (typically 3-4hrs). After growth, the MicroResp plate was read, and final cell count and ATP measurements taken.

From the flow cytometry cell diameter measurements we estimated average cell volumes, and calculated the cellular carbon per cell using

$$
\text { fgC cell }{ }^{-1}=133.754 V^{0.438},
$$

as described by [39]. Multiplying this by the cell counts gives an estimate of the carbon biomass of the culture at the starting and ending points.

The difference between the initial biomass and biomass at the end of the experiment gives the total carbon sequestered through growth. Given an initial biomass $\left(C_{0}\right)$ that grows over time $(t)$ to reach a final biomass $\left(C_{\text {tot }}\right)$, assuming the population is in exponential growth, the mass specific growth rate $(\mu)$ is given by:

$$
\mu=\frac{\log \left(\frac{C_{\mathrm{tot}}}{C_{0}}\right)}{t} .
$$

Respiration rates of cultures were measured during growth using the MicroResp ${ }^{T M}$ system [40]. This is a colorimetric assay initially developed to measure $\mathrm{CO}_{2}$ production from soil samples, which has since been used to measure respiration of bacterial cultures [41, 42, 43]. Briefly, a light sensitive dye is fixed within an agarose gel containing sodium bicarbonate $\left(\mathrm{NaHCO}_{3}\right)$, set into a clear plastic "detection plate". A rubber sealing plate is attached to a deep well plate containing the bacterial cultures and the detection plate clamped into place above, ensuring that the wells are sealed together. A hole in the sealing plate allows $\mathrm{CO}_{2}$ evolved from the culture to reach the detection well, where it reacts with the bicarbonate, changing the $\mathrm{pH}$ of the gel and thereby causing a colour change in the indicator dye. The colour change is measured spectrophotometrically, by measuring the absorbance of the detection plate wells at $572 \mathrm{~nm}$. This is compared to a calibration curve of known $\mathrm{CO}_{2}$ concentrations, so the quantity of $\mathrm{CO}_{2}$ produced by the culture can be determined, and thus the mass (in $\mu \mathrm{g}$ ) of carbon produced calculated. The biomassspecific respiration rate is then calculated using an equation that accounts for changes in biomass of the growing cultures over time [44]:

$$
R=\frac{\mu R_{\mathrm{tot}}}{C_{0} e^{\mu t}-C_{0}}
$$

Here, $R_{\text {tot }}$ is the total mass of carbon produced according to the MicroResp ${ }^{T M}$ measurements, $C_{0}$ is the initial population biomass, $\mu$ is the previously calculated growth rate, and $t$ is the experiment duration.

ATP content of the cultures was measured using the Promega BacTiter-Glo reagent, which produces luminescence in the presence of ATP, proportional to the concentration of ATP. $50 \mu$ l of culture (diluted 1:100) was incubated with $25 \mu \mathrm{l}$ reagent. Luminescence was measured over a 6 minute period to allow the reaction to develop completely, and measurements of luminescence recorded at the $0,2,4$ and 6 minute timepoints. The highest relative light units (RLU) measurement for each culture was taken and used to calculate the quantity of ATP, using

$$
\log (\mathrm{nM} \mathrm{ATP})=1.21 \cdot \log (\mathrm{RLU})-4.69,
$$

derived from a calibration curve. This was then normalised by the flow cytometry measurements to calculate a value of ATP/biomass. This cannot not give a rate of ATP production (as ATP is being both constantly used and produced by the cultures over the duration), but gives snap-shots of the total ATP present in the cultures at given timepoints.

\section{Thermal performance curves}

To quantify thermal performance curves of individual isolates, we fitted the Sharpe-Schoolfield model with the temperature of peak performance $\left(T_{\mathrm{pk}}\right)$ as an explicit parameter $[45,46]$ to the experimentally-derived 
bioRxiv preprint doi: https://doi.org/10.1101/2021.04.14.439774; this version posted April 14, 2021. The copyright holder for this preprint (which was not certified by peer review) is the author/funder, who has granted bioRxiv a license to display the preprint in perpetuity. It is made available under aCC-BY-NC 4.0 International license.

temperature-dependent growth rate and respiration rates of each isolate:

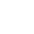

$$
B(T)=B_{0} \frac{e^{\frac{-E}{k} \cdot\left(\frac{1}{T}-\frac{1}{T_{\mathrm{ref}}}\right)}}{1+\frac{E}{E_{\mathrm{D}}-E} e^{\frac{E_{\mathrm{D}}}{k}\left(\frac{1}{T_{\mathrm{pk}}}-\frac{1}{T}\right)}} .
$$

Here, $T$ is temperature in Kelvin (K), $B$ is a biological rate (in this case either growth rate, $\mu$, or respiration rate, $R), B_{0}$ is a temperature-independent metabolic rate constant approximated at some (low) reference temperature $T_{\text {ref }}, E$ is the activation energy in electron volts (eV) (a measure of "thermal sensitivity"), $k$ is the Boltzmann constant $\left(8.617 \times 10^{-5} \mathrm{eV} \mathrm{K}^{-1}\right), T_{\mathrm{pk}}$ is the the temperature where the rate peaks, and $E_{\mathrm{D}}$ the deactivation energy, which determines the rate of decline in the biological rate beyond $T_{\mathrm{pk}}$. We then calculated the peak performance (i.e. $R_{\max }$ or $\mu_{\max }$ ) by solving Eq. 1 for $T=T_{\mathrm{pk}}$. This model was fitted to each dataset using a standard non-linear least squares procedure [44].

The $T_{\mathrm{pk}}$ for growth rate was considered the optimum growth temperature (i.e., $T_{\mathrm{opt}}$ ) for each isolate. Then, the operational niche width was calculated as the difference between $T_{\mathrm{opt}}$ and the temperature below this value where $\mu_{\max }(B(T)$ in eqn 1$)$ reached $50 \%$ of its maximum (i.e, $\mu_{\max }$ at $T_{\mathrm{opt}}$ ). This, a measure of an organism's thermal niche width relevant to typically experienced temperatures $[47,8]$, was used as a quantification of the degree to which an isolate is a thermal generalist or specialist.

\section{Life history trade-offs}

To understand the trade-offs and collinearities between different life history and physiological traits, we performed a principal components analysis $(\mathrm{PCA})$, with optimum growth temperature $\left(T_{\mathrm{opt}}\right)$, niche width, peak growth rate $\left(\mu_{\max }\right)$, peak respiration rate $\left(R_{\max }\right)$, mean cellular ATP content (log-transformed), and carrying capacity $\left(\mathrm{OD}_{600}\right)$ as input variables (scaled to have mean $=0$ and s.d. $=1$ ).

All rate calculations, model fitting and analyses were performed in $\mathrm{R}$.

\section{Phylogenetic reconstruction}

We used $16 \mathrm{~S}$ sequences to build a phylogeny in order to investigate the evolution of thermal performance across the isolated bacterial taxa. Sequences were aligned in MAFFT (v7.205) using the default settings. From this alignment 100 trees were inferred in RAxML (v8.1.1), using a GTR-gamma nucleotide substitution model. The tree with the highest log-likelihood was taken and time-calibrated using PLLDPPDiv, which estimates divergence times using a Dirichlet Process Prior [48]. DPPDiv requires a rooted phylogeny with the nodes in the correct order, however RAxML by default produces an unrooted tree. Therefore, we included an archaeal sequence in our $16 \mathrm{~S}$ alignment (Methanospirillum hungatei, genbank accession NR_074177) and used this as an outgroup in our RAxML run. This gives a tree rooted at the outgroup, which we checked for correct topology using TimeTree (http://www.timetree.org/) as a reference. We derived calibration nodes from TimeTree and performed two DPPDiv runs for 1 million generations each, sampling from the posterior distribution every 100 generations. We ensured that the two runs had converged by verifying that each parameter had an effective sample size above 200 and a potential scale reduction factor below 1.1. We summarised the output of DPPDiv into a single tree using the TreeAnnotator program implemented in BEAST [49]. We then dropped the outgroup tip to give a time-calibrated phylogeny of our $16 \mathrm{~S}$ sequences only, which was used for further analysis.

\section{Phylogenetic trait mapping}

To test whether there was evidence of evolution of $T_{\mathrm{opt}}$, we calculated Pagel's $\lambda$ [50], which quantifies strength of phylogenetic signal - the degree to which shared evolutionary history has driven trait distributions at the tips of a phylogenetic tree. $\lambda=0$ implies no phylogenetic signal, i.e. the signal expected if the trait arose on a star shaped phylogeny. $\lambda=1$ implies strong phylogenetic signal, i.e. that the trait has evolved along the phylogenetic tree (approximated as Brownian motion). Intermediate values $(0<\lambda<1)$ imply deviation from Brownian motion model, an may be observed for different reasons, such as constrained trait evolution due to stabilizing selection, variation in evolutionary rate over time (e.g., due to episodes of rapid niche adaptation).

Pagel's $\lambda$ requires that the trait be normally distributed. However $T_{\text {opt }}$ in our dataset has a right-skewed distribution. Therefore, to test phylogenetic heritability we calculated $\lambda$ for $\log \left(T_{\mathrm{opt}}\right)$.

To map the evolution of $T_{\text {opt }}$ onto our bactrial phylogentic tree, we used maximum likelihood to estimate the ancestral values at each internal node, assuming a Brownian motion model for trait evolution. In 
most cases, $T_{\text {opt }}$ was derived directly from the Sharpe-Schoolfield flow-cytometry growth rate fits. Four strains of Streptomyces proved a special case where the flow-cytometry method did not provide reliable estimates of cell counts (see discussion), so for these strains, growth rates derived from optical density measurements were used to estimate $T_{\mathrm{opt}}$ instead. Finally, for six isolates whose growth was recorded at too few temperatures to fit the Sharpe-Schoolfield model, the temperature with the highest directlymeasured growth rate was taken as an estimate of $T_{\mathrm{opt}}$.

The estimates of phylogenetic signal and the visualisation of trait evolution were performed using tools from the R packages "ape" and "phytools" [51, 52]. The p-value for phylogenetic signal was based on a likelihood ratio test.

\section{Results}

\section{Species sorting}

In total, 74 strains of bacteria were isolated; six from each temperature regime (either incubation followed by isolation at the same temperature (sorting temperature), or incubation followed by isolation at a standard temperature $\left(22^{\circ} \mathrm{C}\right)$ ), with the exception of the $30^{\circ} \mathrm{C}$ sorting temperature regime, from which we obtained 8 isolates. Of these isolates, 62 could be reliably revived in liquid culture, from which 53 grew across a wide enough temperature range to produce enough data points for fitting the Sharpe-Schoolfield model (Eq. 1). The 62 revivable strains represented 14 genera within 3 bacterial phyla (Supplementary Table S1).

Isolates were in general well adapted to their sorting temperature (Fig. 2A). A quadratic linear regression model fitted the data well $(\mathrm{p}<0.0001$, shown in Fig. $2 \mathrm{~A}$ ) and was preferred to a straight-line regression model (ANOVA, $\mathrm{p}<0.0001$ ). The deviation from a simple linear response arises because the $T_{\text {opt }}$ 's of isolates from the three lowest temperatures $\left(4^{\circ} \mathrm{C}, 10^{\circ} \mathrm{C}\right.$, and $\left.21^{\circ} \mathrm{C}\right)$ are significantly higher than their sorting and isolation temperature (Fig. 2A). In comparison, peak growth temperatures of standard temperature isolates are largely unaffected by the temperature that they were previously incubated at (Fig. 2B).

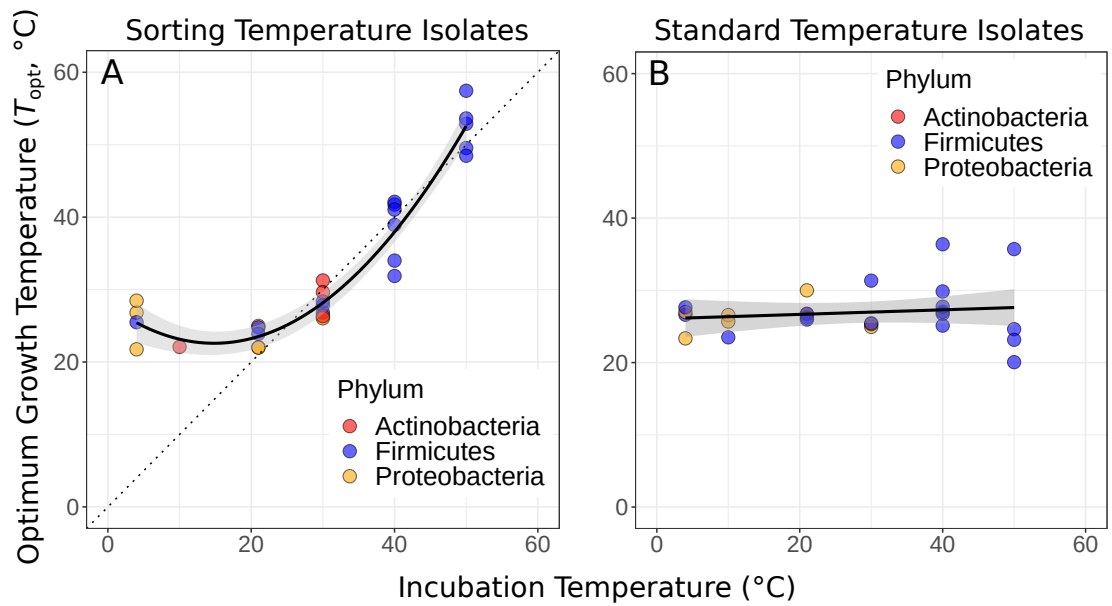

Figure 2: Systematic species sorting of soil bacteria driven by temperature change. A: Thermal optima of growth rate closely match sorting temperature for the isolates from those temperatures (black line: linear quadratic linear regression, $\mathrm{p}<0.0001$ ). Note that the prediction bounds at three lowest temperatures do not overlap with the 1:1 (dashed) line. B: No significant association between incubation temperature and thermal optima for standard temperature isolates (simple linear regression, $\mathrm{p}=0.74$ ). These results show that species sorting can act upon latent diversity to select for isolates adapted to different temperature conditions (A), but that isolates maladapted to the sorting conditions can re-emerge under the appropriate isolation conditions $(\mathbf{B})$.

\section{Evolution of $T_{\text {opt }}$}

$T_{\text {opt }}$ displays a strong signal of phylogenetic heritability, closely approximating a Brownian motion model of trait evolution (Pagel's $\lambda=0.99, \mathrm{p}<0.001$ ), i.e. closely related species have more similar $T_{\mathrm{opt}}$ than 
bioRxiv preprint doi: https://doi.org/10.1101/2021.04.14.439774; this version posted April 14, 2021. The copyright holder for this preprint (which was not certified by peer review) is the author/funder, who has granted bioRxiv a license to display the preprint in perpetuity. It is made available under aCC-BY-NC 4.0 International license.

A

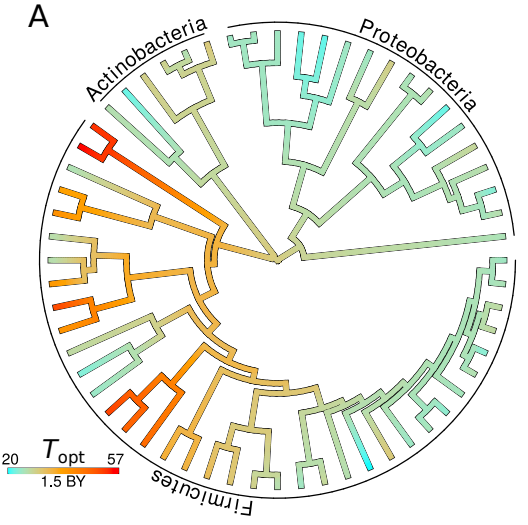

random pairs of species. The estimated ancestral states of $T_{\mathrm{opt}}$ were mapped onto a phylogeny, where it can be seen that colder- or hotter-adapted strains tend to cluster together (Fig. 3A). The estimated evolution of $T_{\text {opt }}$ through time is also shown, where it can seen that a large amount of the trait space is explored by Firmicutes, however Actinobacteria and Proteobacteria are constrained to a much narrower range of optimal growth temperatures (Fig. 3B).

Figure 3: Evolution of $T_{\mathrm{opt}}$. A. Ancestral trait reconstruction of $T_{\mathrm{opt}}$ visualised on a tree, from lower temperatures in cyan, to higher temperatures in red, with time given in billions of years (BY). All of the higher temperature $\left(40-50^{\circ} \mathrm{C}\right)$ isolates belong to the phylum Firmicutes. B. Projection of the phylogenetic tree into the $T_{\mathrm{opt}}$ trait space (y-axis), over relative time (x-axis) since divergence from the root. The clades representative of each phylum are coloured on the projection (Actinobacteria red, Firmicutes blue, Proteobacteria yellow).

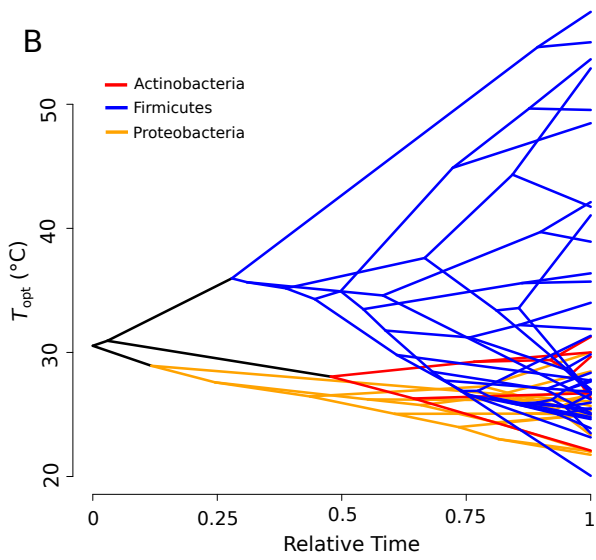

\section{Life-history strategies}

We investigated the level of association and trade-offs between different life history traits in the two major phyla isolated (Firmicutes and Proteobacteria) using PCA (Fig. 4A). Growth specialists (copiotrophs, $r$-specialists) are expected to have high ATP content in combination with high growth rates, but low overall yield (carrying capacity). Yield specialists (oligotrophs, $K$-specialists) should display the opposite pattern, i.e. relatively low growth rates and ATP content, but high yield. The first two principal components explained $60.1 \%$ of the cumulative variation in the data. $T_{\mathrm{opt}}$, carrying capacity and respiration rate showed the largest influence on the first principal component $(\mathrm{PC} 1)$, whilst the growth and niche width traits strongly load onto the second principal component (PC2). The Firmicutes and Proteobacteria phyla are partitioned across these axes. The positive loadings onto $\mathrm{PC} 2$ of growth rate and ATP content versus the negative loading of carrying capacity reveal the $r / K$ trade-off and show that for these isolates, Proteobacteria tend to be $K$-selected whilst Firmicutes tend to be $r$-selected. Furthermore, thermal niche width loads positively onto PC 2 with the growth traits, implying that thermal generalism is not traded off against growth rate here - i.e. the thermal generalists isolated here also tend to have high growth rates.

To further understand the partitioning of taxa into these metabolic strategies, we investigated the differences in accessible cellular energy content (ATP measurements) between these two phyla. We found that across the entire dataset, respiration rate and ATP content display a power-law relationship in both phyla (Fig. 4B). Whilst the Firmicutes have generally higher ATP levels overall, they display a sub-linear scaling relationship of ATP levels with respiration rate (scaling exponent $=0.60 \pm 0.07$ ). In comparison, whist Proteobacteria have less standing ATP content on average, they show an approximately linear scaling relationship between ATP and respiration rate (scaling exponent $=0.99 \pm 0.06$ ). 
bioRxiv preprint doi: https://doi.org/10.1101/2021.04.14.439774; this version posted April 14, 2021. The copyright holder for this preprint (which was not certified by peer review) is the author/funder, who has granted bioRxiv a license to display the preprint in perpetuity. It is made available under aCC-BY-NC 4.0 International license.
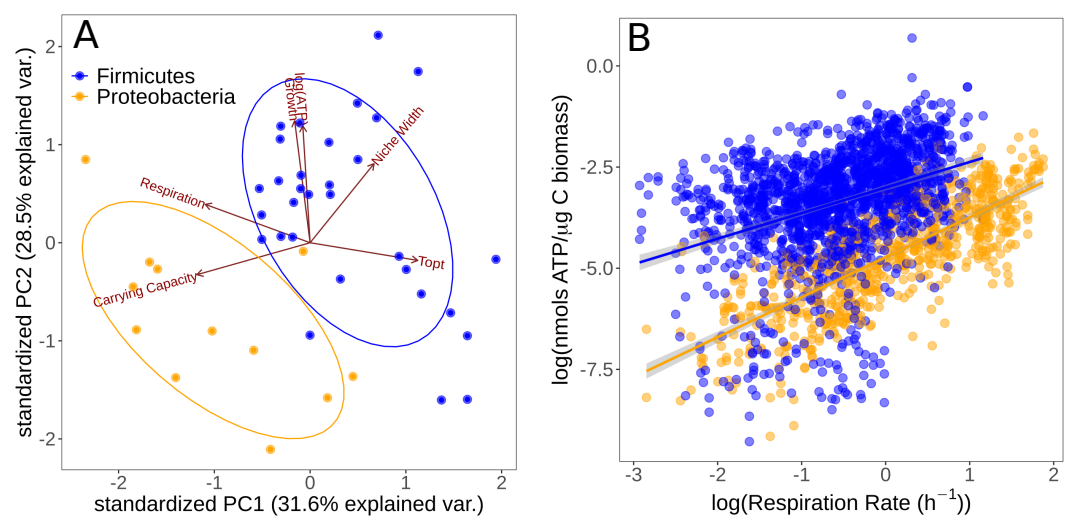

Figure 4: Partitioning of growth strategies between phyla. A: PCA on life-history traits, coloured by phylum. Relative to each other, Firmicutes (blue) tend to be $r$ specialists, Proteobacteria (orange) tend to be $K$ specialists. B: ATP content of cultures is associated with the respiration rate. Firmicutes show a sub-linear scaling relationship of ATP with respiration rate (scaling exponent $=0.60$ \pm 0.07 ), whilst Proteobacteria display an approximately linear scaling relationship (scaling exponent $=$ $0.99 \pm 0.06)$. The same colour scheme is shared by both sub-plots.

\section{Discussion}

Here, we used a species sorting experiment to understand the extent of latent functional diversity present within bacterial communities with respect to temperature fitness. The temperatures treatments resulted in the successful cultivation of bacterial isolates with differing thermal performance. There was a striking similarity between the optimal growth temperatures and isolation temperatures of sorting temperature isolates from the $21^{\circ} \mathrm{C}$ treatment and above (Fig. 2A) showing that, through species sorting on latent diversity, isolation temperature can determine the thermal physiology of taxa cultivated. There was an uneven distribution in the taxa isolated across the temperature range, as well as a significant phylogenetic signature in $T_{\mathrm{opt}}$ (Fig. 3). This indicates that the differences in $T_{\mathrm{opt}}$ were driven by differences in the taxa isolated, rather than adaptation to temperature over the course of the incubation period. Were rapid adaptation during the incubation period a driver for differences in $T_{\mathrm{opt}}$, we would also expect to see a relationship between isolation and incubation temperatures in the standard temperature isolates. This was not the case, with standard temperature isolates showing no correlation between $T_{\text {opt }}$ and their previous incubation temperatures (Fig. 2B). Together, these results show that species sorting processes are likely to have a much greater impact on how communities respond to changing climate, than direct adaptation of microbial strains.

There were differences in the taxa isolated at different temperatures, with more Proteobacteria at lower temperatures and more Firmicutes at higher temperatures (all $T_{\mathrm{opt}}>35^{\circ} \mathrm{C}$ were Firmicutes). These phyla were also partitioned in the trait-space according to the $r / K$ and generalism-specialism trade-offs, with Proteobacteria typified as $K$-selected thermal specialists and Firmicutes as $r$-selected thermal generalists, relative to each other in this study. These findings are not consistent with a specialist-generalist tradeoff in which increasing thermal niche width is proposed to inevitably incur a metabolic cost, reducing maximal growth rates $[36,7]$. However, recent work on phytoplankton thermal performance traits also failed to detect a generalist-specialist trade-off [37], questioning the universality of this potential trade-off. Comparatively increased growth rates and lower respiration rates of Firmicutes relative to Proteobacteria are findings consistent with data from meta-analyses of bacterial rates [53, 3] (cf. Supplementary Fig. S1). Additionally, previously reported values for cellular ATP content have generally been higher for Firmicutes than Proteobacteria with more than 10-fold greater intracellular ATP content reported for Bacillus versus Pseudomonas strains [54], some of the major representatives of Firmicutes and Proteobacteria in this experiment respectively. This suggests that these phyla tend to allocate resources to growth and respiration in fundamentally different ways. One explanation for these seemingly divergent strategies may found in Firmicutes deriving extra energy though fermentation pathways. There is a trade-off between rate and yield whereby bacteria may increase their rate of ATP production through supplementing aerobic respiration with fermentation [55]. This increases the rate of ATP production, at the cost of lower total yield, allowing populations to reach higher growth rates but lower carrying 
bioRxiv preprint doi: https://doi.org/10.1101/2021.04.14.439774; this version posted April 14, 2021. The copyright holder for this preprint (which was not certified by peer review) is the author/funder, who has granted bioRxiv a license to display the preprint in perpetuity. It is made available under aCC-BY-NC 4.0 International license.

capacity from the same resource input. This results in the $r$ vs $K$ selection that we observe here. The differences in scaling relationship between ATP content and respiration rate may provide further evidence of differences in metabolic pathways utilised. Across Proteobacteria, ATP content has a scaling exponent of approximately 1 with respiration rate, indicating that theses strains are deriving ATP solely from aerobic respiration (Fig. 4B). The fact that Firmicutes have a lower scaling exponent $(0.60 \pm 0.07)$ i.e. that they are generating higher levels of ATP than expected at lower rates of respiration, may indicate that they derive ATP from alternative pathways alongside aerobic respiration.

In contrast to the strong association between $T_{\mathrm{opt}}$ and incubation temperature in the sorting temperature isolates, we saw no such relationship for the standard temperature isolates, where mesophiles were consistently recovered regardless of prior incubation conditions. This indicates that species sorting was incomplete (in that maladapted taxa have not gone extinct), implying that bacterial communities can be resilient to temperature change at the community level. Taxa suited to different temperatures are able to "switch on" as as conditions become suitable, allowing community-level functional plasticity due to the latent functional diversity present within communities. Although mesophiles were recovered from all incubation temperatures in our standard temperature experiment, there was the same taxonomic bias as seen in the sorting temperature isolates - more Firmicutes were recovered from higher temperature incubations. This is a reflection of the propensity of these bacteria to form endospores and remain dormant until conditions are favourable, upon which they invest resources into rapid growth to gain a competitive advantage over other taxa, consistent with our life-history trait findings of $r$-specialism in Firmicutes. In comparison, the Proteobacteria in this experiment were generally more suited to oligotrophic environments (e.g. Collimonas, [56]), where constituent species are expected to present low growth rate and high carrying capacity ( $K$ specialists, [57]) as well as increased respiration [58]. This idea is reinforced by the fact that the soil from which these strains were isolated from is sandy and acidic (i.e. oligotrophic) [59] and sequencing studies reveal Proteobacteria as the most abundant phylum [60]. We do not suggest that this $r$ vs $K$ specialism is general to all Firmicutes or Proteobacteria, indeed meta-analysis reveals little consistency in the phyla associated with copiotrophy or oligotrophy [61]. Nor do we suggest that warming is likely to result in selection for Firmicutes over Proteobacteria - community temperature responses are not likely to be consistent at coarse phylogenetic levels [1]. However, the results presented here are consistent with phylum-specific traits for the majority of our isolates, when compared to each other.

Patterns of succession reflect these differences in growth strategy between taxa. Studies have revealed taxonomic groups associated with different stages of microbial succession, with patterns broadly consistent across timescales of days $[62,63,14]$ and years $[64,65]$ through to upwards of thousands and tens of thousands of years, as revealed through sequencing of soil sediments [66]. Generally, across these studies the phyla Firmicutes and Bacteroidetes are associated with early succession, whilst other phyla such as Actinobacteria and Acidobacteria are more abundant at later stages of succession. Proteobacteria are less consistent at the phylum level, with Alphaproteobacteria associated with late succession, Betaproteobacteria associated with early succession and Gammaproteobacteria variously associated with different stages of succession in different studies. Isolated taxa reveal a strong association between early succession and high growth rates [63] as well as rRNA operon copy numbers, a key determinant of bacterial growth rate [67]. The $K$-selected taxa may therefore be thought of as general constituents of soil, associated with standard low turnover of carbon, whilst the $r$-selected taxa may been seen as more opportunistic from their involvement in early succession. Indeed, signatures of community-level differences in $r$ - versus $K$ selection have been observed in microbial communities at different successional stages [68]. Fluctuating temperatures may therefore drive repeated assembly dynamics via sorting on latent microbial diversity, leading to functional community changes through time.

Amongst the higher temperature isolates were obligate thermophiles; the lowest growth temperature that was recorded for the $50^{\circ} \mathrm{C}$ incubation temperature isolated strains was $35^{\circ} \mathrm{C}$. As the growth of these strains is constrained to temperatures largely outside the range experienced by the soils they were isolated from, we conclude that these species are present due to dispersal and are not active members of the soil community. In comparison, no obligate psychrophiles were isolated in this experiment; the lowest incubation temperature isolates still displayed $T_{\text {opt }}$ 's in the $20-25^{\circ} \mathrm{C}$ range (Figs. $2 \mathrm{~A}$ and 3 ). The sampling was carried out in June, when ambient soil surface temperature was $19.4^{\circ} \mathrm{C}$. Therefore it is possible that only the "summer community" was sampled and that psychrophiles may be found were the sampling to be carried out in winter under colder ambient conditions. However, it may be simply that true psychrophiles are either not present in the soil in sufficient quantities to be reliably isolated, or tend to be adapted to such specific environments that lab isolation is unreliable. This is reflected in thermal performance data from bacteria isolated from very cold environments (e.g. Arctic or Antarctic samples), where growth rate is often seen to be optimal at more mesophilic temperatures $\left(\sim 20^{\circ} \mathrm{C}\right)$ 
$[69,70]$. Furthermore, strains routinely cultured at low temperatures have been shown to often display higher peak growth temperatures than may be expected [3].

In summary, we have found that latent functional diversity activated by phenotypically plastic responses to temperature change can allow the thermal fitness of bacteria to track dramatic changes in temperature at the community level. Community function is expected to be driven by interactions between the most abundant taxa [71] and therefore changes in the abundance of taxa with temperature change are likely to drive profound changes in overall community function. In particular, $r$ - vs $K$ - selection is likely to vary with temperature change at the community level, from daily to seasonal successional trajectories, driven by species sorting. Furthermore, climate change is expected to lead to increased temperature fluctuations [72], both in magnitude and frequency. This may potentially lead to more frequent species sorting effects over short timescales, further driving changes in community composition through time. Overall, these results show that latent diversity in thermal physiology combined with temperature induced species sorting is likely to facilitate the responses of microbial community structure and functioning to climatic fluctuations.

\section{Acknowledgments}

TPS was supported by a BBSRC DTP scholarship (BB/J014575/1). DGK was supported by a NERC DTP scholarship (NE/L002515/1) TB and SP were funded by NERC grants NE/M020843/1 and NE/S000348/1.

\section{References}

[1] Oliverio AM, Bradford MA, Fierer N. Identifying the microbial taxa that consistently respond to soil warming across time and space. Global Change Biology. 2017;23(5):2117-2129. doi:10.1111/gcb.13557.

[2] García FC, Bestion E, Warfield R, Yvon-Durocher G. Changes in temperature alter the relationship between biodiversity and ecosystem functioning. Proceedings of the National Academy of Sciences of the United States of America. 2018;115(43):10989-10994. doi:10.1073/pnas.1805518115.

[3] Smith TP, Thomas TJH, Garcia-Carreras B, Sal S, Yvon-Durocher G, Bell T, et al. Communitylevel respiration of prokaryotic microbes may rise with global warming. Nature Communications. 2019;10:5124. doi:10.1038/s41467-019-13109-1.

[4] Bardgett RD, Freeman C, Ostle NJ. Microbial contributions to climate change through carbon cycle feedbacks. ISME Journal. 2008;2(8):805-814. doi:10.1038/ismej.2008.58.

[5] Corkrey R, McMeekin TA, Bowman JP, Ratkowsky DA, Olley J, Ross T. The Biokinetic Spectrum for Temperature. Plos One. 2016;11(4):e0153343. doi:10.1371/journal.pone.0153343.

[6] Huey RB, Kingsolver JG. Evolution of Thermal Sensitivity of Ectotherm Performance. Trends in ecology \& evolution. 1989;4(5):131-135.

[7] Angilletta Jr MJ. Thermal acclimation. In: Thermal Adaption: A theoretical and empirical synthesis; 2009. p. 126-156.

[8] Kontopoulos DG, Smith TP, Barraclough TG, Pawar S. Adaptive evolution shapes the present-day distribution of the thermal sensitivity of population growth rate. PLOS Biology. 2020;18(10):e3000894. doi:10.1371/journal.pbio.3000894.

[9] Pucci F, Rooman M. Physical and molecular bases of protein thermal stability and cold adaptation. Current Opinion in Structural Biology. 2017;42:117-128. doi:10.1016/j.sbi.2016.12.007.

[10] Bennett AF, Dao KM, Lenski RE. Rapid evolution in response to high-temperature selection. Nature. 1990;346(6279):79-81. doi:10.1038/346079a0.

[11] Kishimoto T, Iijima L, Tatsumi M, Ono N, Oyake A, Hashimoto T, et al. Transition from positive to neutral in mutation fixation along with continuing rising fitness in thermal adaptive evolution. PLoS Genetics. 2010;6(10). doi:10.1371/journal.pgen.1001164. 
[12] Blaby IK, Lyons BJ, Wroclawska-Hughes E, Phillips GCF, Pyle TP, Chamberlin SG, et al. Experimental evolution of a facultative thermophile from a mesophilic ancestor. Applied and Environmental Microbiology. 2012;78(1):144-155. doi:10.1128/AEM.05773-11.

[13] Scheuerl T, Hopkins M, Nowell RW, Rivett DW, Barraclough TG, Bell T. Bacterial adaptation is constrained in complex communities. Nature Communications. 2020;11(754). doi:10.1038/s41467020-14570-z.

[14] Rui J, Peng J, Lu Y. Succession of bacterial populations during plant residue decomposition in rice field soil. Applied and Environmental Microbiology. 2009;75(14):4879-4886. doi:10.1128/AEM.00702-09.

[15] Suutari M, Laakso S. Microbial fatty acids and thermal adaptation. vol. 20; 1994.

[16] Luo Y, Wan S, Hui D, Wallace LL. Acclimatization of soil respiration to warming in a tall grass prairie. Nature. 2001;413(6856):622-625. doi:10.1038/35098065.

[17] Bradford MA. Thermal adaptation of decomposer communities in warming soils. Frontiers in Microbiology. 2013;4(NOV):1-16. doi:10.3389/fmicb.2013.00333.

[18] Karhu K, Auffret MD, Dungait JAJ, Hopkins DW, Prosser JI, Singh BK, et al. Temperature sensitivity of soil respiration rates enhanced by microbial community response. Nature. 2014;513(7516):8184. doi:10.1038/nature13604.

[19] Melillo JM, Frey SD, DeAngelis KM, Werner WJ, Bernard MJ, Bowles FP, et al. Long-term pattern and magnitude of soil carbon feedback to the climate system in a warming world. Science. 2017;358(6359):101-105. doi:10.1126/science.aan2874.

[20] Allison SD, Martiny JBH. Resistance, resilience, and redundancy in microbial communities. Proceedings of the National Academy of Sciences. 2008;105:11512-11519. doi:10.17226/12501.

[21] Aydogan EL, Moser G, Müller C, Kämpfer P, Glaeser SP. Long-term warming shifts the composition of bacterial communities in the phyllosphere of Galium album in a permanent grassland field-experiment. Frontiers in Microbiology. 2018;9. doi:10.3389/fmicb.2018.00144.

[22] Balser TC, Firestone MK. Linking microbial community composition and soil processes in a California annual grassland and mixed-conifer forest. Biogeochemistry. 2005;73(2):395-415. doi:10.1007/s10533-004-0372-y.

[23] Yu H, Deng Y, He Z, Van Nostrand JD, Wang S, Jin D, et al. Elevated CO2 and warming altered grassland microbial communities in soil top-layers. Frontiers in Microbiology. 2018;9(AUG):1-10. doi:10.3389/fmicb.2018.01790.

[24] Van der Gucht K, Cottenie K, Muylaert K, Vloemans N, Cousin S, Declerck S, et al. The power of species sorting: Local factors drive bacterial community composition over a wide range of spatial scales. Proceedings of the National Academy of Sciences. 2007;104(51):20404-20409. doi:10.1073/pnas.0707200104.

[25] Langenheder S, Székely AJ. Species sorting and neutral processes are both important during the initial assembly of bacterial communities. ISME Journal. 2011;5(7):1086-1094. doi:10.1038/ismej.2010.207.

[26] Székely AJ, Langenheder S. The importance of species sorting differs between habitat generalists and specialists in bacterial communities. FEMS Microbiology Ecology. 2014;87(1):102-112. doi:10.1111/1574-6941.12195.

[27] Curtis TP, Sloan WT, Scannell JW. Estimating prokaryotic diversity and its limits. Proceedings of the National Academy of Sciences. 2002;99(16):10494-10499. doi:10.1073/pnas.142680199.

[28] Sogin ML, Morrison HG, Huber JA, Welch DM, Huse SM, Neal PR, et al. Microbial Diversity in the Deep Sea and the Underexplored "Rare Biosphere". PNAS. 2006;103(32):12115-12120. doi:10.1073/pnas.0605127103.

[29] Lynch MDJ, Neufeld JD. Ecology and exploration of the rare biosphere. Nature Reviews Microbiology. 2015;13(4):217-229. doi:10.1038/nrmicro3400. 
[30] Marchant R, Franzetti A, Pavlostathis SG, Tas DO, Erdbrugger I, Unyayar A, et al. Thermophilic bacteria in cool temperate soils: Are they metabolically active or continually added by global atmospheric transport? Applied Microbiology and Biotechnology. 2008;78(5):841-852. doi:10.1007/s00253-008-1372-y.

[31] Hubert C, Loy A, Nickel M, Arnosti C, Baranyi C, Brüchert V, et al. A constant flux of diverse thermophilic bacteria into the cold arctic seabed. Science. 2009;325(5947):1541-1544. doi:10.1126/science.1174012.

[32] Zeigler DR. The Geobacillus paradox: Why is a thermophilic bacterial genus so prevalent on a mesophilic planet? Microbiology (United Kingdom). 2014;160(PART 1):1-11. doi:10.1099/mic.0.071696-0.

[33] Gilchrist GW. Specialists and generalists in changing environments. I. Fitness landscapes of thermal sensitivity. American Naturalist. 1995;146(2):252-270. doi:10.2307/2678832.

[34] Basan M, Honda T, Christodoulou D, Hörl M, Chang YF, Leoncini E, et al. A universal trade-off between growth and lag in fluctuating environments. Nature. 2020;584(April 2018). doi:10.1038/s41586020-2505-4.

[35] Seebacher F, Ducret V, Little AG, Adriaenssens B. Generalist - specialist trade-off during thermal acclimation. Royal Scociety Open Science. 2015;2:140251. doi:10.1098/rsos.140251.

[36] Huey RB, Hertz PE. Is a Jack-of-All-Temperatures a Master of None? Evolution. 1984;38(2):441. doi: $10.2307 / 2408502$.

[37] Kontopoulos DG, Yvon-durocher G, Sebille EV, Lange M, Barraclough TG, Pawar S. Phytoplankton thermal responses adapt in the absence of hard thermodynamic constraints. Evolution. 2020;doi:10.1111/evo.13946.

[38] Andrews JH, Harris RF. r- and K-Selection and Microbial Ecology. 1986; p. 99-147. doi:10.1007/9781-4757-0611-6_3.

[39] Romanova ND, Sazhin AF. Relationships between the cell volume and the carbon content of bacteria. Oceanology. 2010;50(4):522-530. doi:10.1134/S0001437010040089.

[40] Campbell CD, Chapman SJ, Cameron CM, Davidson MS, Potts JM. A rapid microtiter plate method to measure carbon dioxide evolved from carbon substrate amendments so as to determine the physiological profiles of soil microbial communities by using whole soil. Applied and Environmental Microbiology. 2003;69(6):3593-3599. doi:10.1128/AEM.69.6.3593.

[41] Lawrence D, Fiegna F, Behrends V, Bundy JG, Phillimore AB, Bell T, et al. Species interactions alter evolutionary responses to a novel environment. PLoS Biology. 2012;10(5). doi:10.1371/journal.pbio.1001330.

[42] Foster KR, Bell T. Competition, not cooperation, dominates interactions among culturable microbial species. Current biology. 2012;22(19):1845-50. doi:10.1016/j.cub.2012.08.005.

[43] Rivett DW, Lilley AK, Connett GJ, Carroll MP, Legg JP, Bruce KD. Contributions of Composition and Interactions to Bacterial Respiration Are Reliant on the Phylogenetic Similarity of the Measured Community. Microbial Ecology. 2017;74(3):757-760. doi:10.1007/s00248-017-0982-2.

[44] Smith TP, Clegg T, Bell T, Pawar S. Systematic variation in the temperature dependence of bacterial carbon use efficiency. bioRxiv. 2020;doi:10.1101/2020.09.14.296095.

[45] Schoolfield RM, Sharpe PJ, Magnuson CE. Non-linear regression of biological temperaturedependent rate models based on absolute reaction-rate theory. Journal of theoretical biology. 1981;88(4):719-31.

[46] Kontopoulos DG, van Sebille E, Lange M, Yvon-Durocher G, Barraclough TG, Pawar S. Phytoplankton thermal responses adapt in the absence of hard thermodynamic constraints. bioRxiv. 2018;doi:10.1101/452250.

[47] Pawar S, Dell AI, Savage VM, Knies JL. Real versus Artificial Variation in the Thermal Sensitivity of Biological Traits. The American Naturalist. 2016;187(2). doi:10.1086/684590. 
[48] Heath TA, Holder MT, Huelsenbeck JP. A dirichlet process prior for estimating lineage-specific substitution rates. Molecular Biology and Evolution. 2012;29(3):939-955. doi:10.1093/molbev/msr255.

[49] Bouckaert R, Vaughan TG, Barido-Sottani J, Duchêne S, Fourment M, Gavryushkina A, et al. BEAST 2.5: An advanced software platform for Bayesian evolutionary analysis. PLoS Computational Biology. 2019;15(4):1-28. doi:10.1371/journal.pcbi.1006650.

[50] Pagel M. Inferring historical patterns of biological evolution. Nature. 1999;401:877-884.

[51] Revell LJ. phytools: An R package for phylogenetic comparative biology (and other things). Methods in Ecology and Evolution. 2012;3(2):217-223. doi:10.1111/j.2041-210X.2011.00169.x.

[52] Revell LJ. Two new graphical methods for mapping trait evolution on phylogenies. Methods in Ecology and Evolution. 2013;4(8):754-759. doi:10.1111/2041-210X.12066.

[53] Makarieva AM, Gorshkov VG, Li BL. Energetics of the smallest: do bacteria breathe at the same rate as whales? Proceedings of the Royal Society B: Biological Sciences. 2005;272(1577):2219-2224. doi:10.1098/rspb.2005.3225.

[54] Hattori N, Sakakibara T, Kajiyama N, Igarashi T, Maeda M, Murakami S. Enhanced microbial biomass assay using mutant luciferase resistant to benzalkonium chloride. Analytical Biochemistry. 2003;319(2):287-295. doi:10.1016/S0003-2697(03)00322-1.

[55] Pfeiffer T, Schuster S, Bonhoeffer S. Cooperation and competition in the evolution of ATP-producing pathways. Science. 2001;292(5516):504-507. doi:10.1126/science.1058079.

[56] Leveau JHJ, Uroz S, de Boer W. The bacterial genus Collimonas: Mycophagy, weathering and other adaptive solutions to life in oligotrophic soil environments. Environmental Microbiology. 2010;12(2):281-292. doi:10.1111/j.1462-2920.2009.02010.x.

[57] Fierer N, Bradford MA, Jackson RB. Toward an Ecological Classification of Soil Bacteria. Ecology. 2007;88(6):1354-1364.

[58] Keiblinger KM, Hall EK, Wanek W, Szukics U, Hämmerle I, Ellersdorfer G, et al. The effect of resource quantity and resource stoichiometry on microbial carbon-use-efficiency. FEMS Microbiology Ecology. 2010;73(3):430-440. doi:10.1111/j.1574-6941.2010.00912.x.

[59] Fornara DA, Banin L, Crawley MJ. Multi-nutrient vs. nitrogen-only effects on carbon sequestration in grassland soils. Global Change Biology. 2013;19(12):3848-3857. doi:10.1111/gcb.12323.

[60] Macdonald CA, Crawley MJ, Wright DJ, Kuczynski J, Robinson L, Knight R, et al. Identifying qualitative effects of different grazing types on below-ground communities and function in a long-term field experiment. Environmental Microbiology. 2015;17(3):841-854. doi:10.1111/1462-2920.12539.

[61] Ho A, Di Lonardo DP, Bodelier PLE. Revisiting life strategy concepts in environmental microbial ecology. FEMS microbiology ecology. 2017;93(3):1-14. doi:10.1093/femsec/fix006.

[62] Noll M, Matthies D, Frenzel P, Derakshani M, Liesack W. Succession of bacterial community structure and diversity in a paddy soil oxygen gradient. Environmental Microbiology. 2005;7(3):382395. doi:10.1111/j.1462-2920.2004.00700.x.

[63] Shrestha PM, Noll M, Liesack W. Phylogenetic identity, growth-response time and rRNA operon copy number of soil bacteria indicate different stages of community succession. Environmental Microbiology. 2007;9(10):2464-2474. doi:10.1111/j.1462-2920.2007.01364.x.

[64] Nemergut DR, Anderson SP, Cleveland CC, Martin AP, Miller AE, Seimon A, et al. Microbial community succession in an unvegetated, recently deglaciated soil. Microbial Ecology. 2007;53(1):110122. doi:10.1007/s00248-006-9144-7.

[65] Banning NC, Gleeson DB, Grigg AH, Grant CD, Andersen GL, Brodie EL, et al. Soil microbial community successional patterns during forest ecosystem restoration. Applied and Environmental Microbiology. 2011;77(17):6158-6164. doi:10.1128/AEM.00764-11.

[66] Jangid K, Whitman WB, Condron LM, Turner BL, Williams MA. Soil bacterial community succession during long-term ecosystem development. Molecular Ecology. 2013;22(12):3415-3424. doi: $10.1111 /$ mec. 12325 . 
[67] Klappenbach J, Dunbar JM, Schmidt TM. rRNA gene copy number reflects ecological strategies in bacteria. Applied and Environmental Microbiology. 2000;66(4):1328-1333. doi:10.1128/AEM.66.4.1328-1333.2000.

[68] Pascual-García A, Bell T. Community-level signatures of ecological succession in natural bacterial communities. Nature Communications. 2020;11(1):1-11. doi:10.1038/s41467-020-16011-3.

[69] Bowman JP, Gosinkt JJ, Mccammon Sa, Skerratt H, Staley JT, Mcmeekinn Ta. Colwellia demingiae sp. nov., Colwellia hornerae sp. nov., Colwellia rossensis sp. nov. and Colwellia psychrotropica sp. nov.: psychrophilic Antarctic species with the ability to synthesize docosahexaenoic acid (22:6 3$)$. International Journal of Systematic Bacteriology. 1998;(48):1171-1180.

[70] Knoblauch C, Jorgensen BB. Effect of temperature on sulphate reduction, growth rate and growth yield in five psychrophilic sulphate-reducing bacteria from Arctic sediments. Environmental Microbiology. 1999;1(5):457-467. doi:10.1046/j.1462-2920.1999.00061.x.

[71] Rivett DW, Bell T. Abundance determines the functional role of bacterial phylotypes in complex communities. Nature Microbiology. 2018;3(7):767-772. doi:10.1038/s41564-018-0180-0.

[72] Vasseur Da, Delong JP, Gilbert B, Greig HS, Harley CDG, Mccann KS, et al. Increased temperature variation poses a greater risk to species than climate warming Increased temperature variation poses a greater risk to species than climate warming. Proceedings of the Royal Society B. 2014;281. doi:10.1098/rspb.2013.2612. 